\title{
ACOLHIMENTO COM AVALIAÇÃO E CLASSIFICAÇÃO DE RISCO: A ÓPTICA DOS FUTUROS ENFERMEIROS
}

\author{
EMBRACEMENT WITH ASSESSMENT AND HOME RISK RATING: THE \\ PERSPECTIVE OF FUTURE NURSES
}

\section{ACOGIMIENTO CON LA EVALUACIÓN Y CLASIFICACIÓN DE RIESGOS: LA PERSPECTIVA DE LAS FUTURAS ENFERMERAS}

\author{
Maicon de Araújo Nogueira ${ }^{1}$, Danielle Oliveira $\mathrm{Maciel}^{2}$, João Vitor de Oliveira Ribeiro ${ }^{3}$, \\ Márcio Almeida Lins ${ }^{4}$, Antônia Margareth Moita Sá ${ }^{5}$
}

\section{RESUMO}

Objetivo: descrever o saber de um grupo de acadêmicos do Curso de Graduação em Enfermagem de uma Instituição de Ensino Superior (IES) pública de Belém-PA, sobre o processo de acolhimento com avaliação e classificação de risco. Método: Trata-se de pesquisa descritiva, exploratória com abordagem qualitativa. A coleta de dados ocorreu por meio de entrevista semi-estruturada, cujo instrumento foi um roteiro de entrevista elaborado com perguntas abertas. As falas dos 16 acadêmicos, de ambos os gêneros, foram gravadas e em seguida transcritas e analisadas. Resultados: Foi identificado o distanciamento entre o que é ensinado no Curso de Graduação em Enfermagem e aquilo que o profissional vivencia em sua prática. Conclusão: Existem avanços inquestionáveis em relação à inclusão do tema na Graduação em Enfermagem. Atenta-se para as fragilidades evidenciadas nas dimensões estruturais e recursos humanos, uma vez que, provavelmente, interfiram negativamente na construção de conhecimentos sólidos acerca da temática.

Descritores: Acolhimento; Classificação; Educação em Enfermagem.

\section{ABSTRACT}

Objective: describe the knowledge of a group of students of the undergraduate course in Nursing of a public higher education institution of Belém-PA about the process of embracement with assessment and home risk rating. Method: This is a descriptive, exploratory qualitative approach. The data were collected through semi-structured interview, which used an interview guide prepared with open questions. The statements of 16 students, $o$ both sexes, were recorded and transcribed and analyzed then. Results: the gap has been identified between what is taught in the Undergraduate Course in Nursing and what professional experiences in their practice. Conclusion: There are unquestionable advances in relation to the inclusion of the topic in undergraduate nursing. It is attentive to the weaknesses evidenced in the structural dimensions and human resources, since they will probably interfere negatively in the construction of solid knowledge about the subject.

Descriptors: User Embracement; Classification; Education; Nursing.

1Enfermeiro. Especialista em Urgência e Emergência. Docente da Faculdade Metropolitana da AmazôniaFAMAZ, Belém, PA, Brasil. E-mail: enfnogueira@globomail.com

2Enfermeira. Universidade do Estado Pará-UEPA. Belém, PA, Brasil. E-mail: oliveira.danimac@gmail.com 3Enfermeiro. Universidade do Estado Pará-UEPA. Belém, PA, Brasil. E-mail: oliveira.ribeiro75@gmail.com 4Acadêmico de enfermagem. Faculdade Metropolitana da Amazônia-FAMAZ, Belém, PA, Brasil. E-mail: enflins@gmail.com

5Enfermeira pela Universidade Federal do Pará, mestrado em Enfermagem pela Universidade Federal do Rio de Janeiro, doutorado em Enfermagem pela Universidade Federal do Rio de Janeiro. Professora adjunto IV da Universidade do Estado do Pará, e enfermeira assistente - Secretaria de Estado de Saúde Pública. 


\section{RESUMEN}

Objetivo: Describir el conocimiento de un grupo de estudiantes del curso de licenciatura en enfermería de una institución de educación superior pública de Belém-PA en el proceso de host con la evaluación del riesgo y la clasificación. Método: Se trata de un enfoque cualitativo descriptivo, exploratorio. Los datos fueron recolectados a través de entrevista semiestructurada, que utiliza una guía de entrevista preparado con preguntas abiertas. Las declaraciones de 16 académicos, de ambos sexos, fueron grabadas y transcritas y analizadas a continuación. Resultados: la brecha se ha identificado entre lo que se enseña en el Curso de Licenciatura en Enfermería y qué experiencias profesionales en su práctica. Conclusión: Hay indudables avances en relación con la inclusión del tema en el de graduación. Atento a las deficiencias puestas de manifiesto en las dimensiones estructurales y de recursos humanos, ya que, probablemente, un impacto negativo en la construcción de conocimientos sobre el tema.

Descriptores: Acogimiento; Clasificación; Educación en Enfermería.

\section{INTRODUÇÃO}

A área de urgência e emergência é um importante componente da assistência à saúde. Nos últimos anos, houve um aumento da demanda por esses serviços, decorrente do crescimento do número de acidentes, da violência urbana e da falta de estrutura da atenção básica. ${ }^{1}$

O Serviço Hospitalar de Emergência (SHE) tem como finalidade atender pessoas com comprometimento grave de saúde e acolher casos não urgentes para direcioná-los a outros serviços de menor complexidade. Desse modo, o pressuposto fundamental dos SHE é a garantia de acesso e acolhimento às pessoas com necessidades emergenciais de saúde, de forma organizada, a fim de prevenir iatropatogenias por manipulação ou tratamentos incorretos, evitando-se morte ou incapacidade física, seja esta temporária ou permanente. ${ }^{2}$

O enfermeiro é o profissional mais indicado para desempenhar esta função, considerando que a triagem não é de caráter diagnóstico e sim de identificação de prioridades no atendimento. ${ }^{3} \mathrm{~A}$ triagem classificatória de risco envolve um complexo processo de tomada de decisão e, por isso, diferentes sistemas têm sido desenvolvidos no mundo todo, para auxiliar os enfermeiros na classificação de gravidade mais adequada a cada caso. De modo geral, esses sistemas de classificação visam diminuir o tempo de espera do paciente no serviço hospitalar de emergência, por meio da priorização do atendimento dos casos de maior gravidade, cujo prognóstico tende a ser mais sombrio com o atraso do início do tratamento. ${ }^{4}$ 
A falta de profissionais com o devido conhecimento sobre a finalidade e funcionamento dos setores de acolhimento com classificação de risco, se revestem de grande importância, sendo uma das maiores dificuldades encontradas, para que haja um melhor andamento deste setor. 5,6

Para desenvolver o acolhimento com classificação de risco são necessárias três habilidades fundamentais: avaliação, conhecimento e intuição. Estas habilidades se desenvolvem com a experiência, o que para um recém graduado é bem difícil, somado ao déficit de conhecimento acerca dos processos que envolvem a atividade. ${ }^{7}$

A literatura aponta para um déficit de conhecimento dos acadêmicos de enfermagem, o que se deve, ao pouco ou nenhum contato que os mesmos têm com esses temas durante a formação. Os profissionais de enfermagem precisam estar capacitados para assistir de forma integral e qualitativa o paciente, entretanto, para que essa assistência seja de qualidade é necessário que o acadêmico, futuro profissional, seja preparado desde a graduação. ${ }^{8}$

O estudo justifica-se pelo fato da literatura apontar entraves no processo, e dentre estes entraves destaca-se as inadequações na estrutura curricular das instituições formadoras, afetando diretamente na formação de profissionais e consequentemente na assistência prestada. Dessa forma, surgiram os seguintes questionamentos: Dada a importância que o profissional enfermeiro tem no processo de acolhimento com avaliação e classificação de risco, qual a visão que os futuros enfermeiros possuem acerca desse processo? Esses acadêmicos sentem-se preparados para desenvolver essa atividade? Em que momento eles têm contato com o tema? E qual o papel da academia para a obtenção deste conhecimento?

Para responder aos questionamentos propôs-se a realização deste estudo, objetivando descrever o saber de um grupo de acadêmicos do Curso de Graduação em Enfermagem de uma Instituição de Ensino Superior (IES) pública de Belém-Pa, sobre o processo de acolhimento com avaliação e classificação de risco.

\section{MÉTODO}

Trata-se de uma pesquisa descritiva, exploratória, com abordagem qualitativa. ${ }^{9}$ Participaram da pesquisa acadêmicos do curso de graduação em enfermagem de uma Instituição de Ensino Superior (IES) 
pública de Belém-PA, devidamente matriculados no $5^{\circ}$ ano do curso, nos turnos matutino e vespertino, de ambos os gêneros, no período de fevereiro à março de 2015, até saturação em torno dos eixos temáticos. ${ }^{10}$ Do universo de 74 discentes no setor de registro acadêmico da instituição, somente 16 participaram da pesquisa.

Pesquisa foi aprovada pelo Comitê de Ética em Pesquisa (CEP), do Curso de Graduação em Enfermagem da Universidade do Estado do Pará (UEPA), CAAE: 39511314.0.0000.5170, número do parecer: 946.515, com autorização prévia da instituição e posterior assinatura do Termo de Consentimento Livre e Esclarecido (TCLE) pelos participantes da pesquisa.

Para a coleta de dados utilizou-se como técnica a entrevista semi-estruturada, tendo como instrumento para a coleta um roteiro de entrevista elaborado com perguntas abertas permitindo que os entrevistados pudessem discorrer acerca dos questionamentos sem tempo prédeterminado para as respostas. As falas dos entrevistados foram gravadas, e em seguida transcritas na íntegra para o software Microsoft Office Word 2010 do
Windows. Após a transcrição, realizou-se leitura criteriosa seguindo-se os momentos metodológicos propostos por Bardin ${ }^{11,12}$ sendo criadas categorias empíricas as quais foram discriminadas por tema e descritas. As falas dos entrevistados foram identificadas por códigos alfanuméricos, objetivando preservar o anonimato dos participantes, utilizando-se as seguintes denominações: “ACD 1", “ACD 2", “ACD 3...” respectivamente. Nas convergências das unidades de registro, realizou-se a montagem e agrupamento das falas dos entrevistados por similaridade de conteúdo temático.

A pesquisa foi realizada seguindo os pressupostos que regulamentam pesquisa envolvendo seres humanos contidos na resolução no 466/ 2012 do CNS.

\section{RESULTADOS E DISCUSSÃO}

Os dados coletados foram submetidos à técnica de análise de conteúdo temático, na qual o tema é a unidade de significação que se liberta naturalmente de um texto analisado e nessa fase de interpretação $\operatorname{dos} \operatorname{dados} \quad \mathrm{o}$ pesquisador necessita retornar ao referencial teórico na busca do embasamento das análises fornecendo sentido à interpretação. ${ }^{11}$ 
$\mathrm{O}$ corpus do estudo possibilitou a organização do conteúdo em quatro categorias empíricas, agrupadas de acordo com o tema extraído das respostas.

\section{PRIMEIRA CATEGORIA:}

ENTENDIMENTO ACERCA DO PROCESSO DE ACOLHIMENTO COM AVALIAÇÃO E CLASSIFICAÇÃO DE RISCO:

É possível verificar que o entendimento dos pesquisados acerca do acolhimento com classificação de risco limita-se a um meio de priorizar o atendimento sem estar vinculado ao acolhimento dos usuários, divergindo da proposta do Ministério da Saúde, como podemos observar nas falas:

É a questão que você classifica o paciente de acordo com a necessidade que ele tem, por exemplo, o paciente que tem a cor vermelha ele é urgente, tem que atender logo; aquele paciente amarelinho pode esperar um pouco mais; laranja, verde, azul. (ACD 3)

[...] tem aquela classificação de Manchester né, a classificação de cores, que é pra priorizar o atendimento, quem é urgente, quem corre risco de morrer, e o que não tem, que vai do vermelho até o verde. Acho que é isso. (ACD 5)

O Acolhimento com Classificação de Risco possui outros objetivos além de organizar as filas de espera e propor uma ordem de atendimento que não a ordem de chegada, como por exemplo: garantir atendimento imediato e humanizado às pessoas que chegam nas portas dos serviços de urgência com risco elevado, dar informar aos pacientes e seus familiares, aumentar a satisfação dos usuários e possibilitar construção de redes internas e externas do atendimento. ${ }^{13}$

Observou-se que alguns pesquisados não têm definição clara do protocolo e fazem confusão na descrição das cores quando relacionadas aos critérios de urgência e emergência, como observamos nas falas seguintes:

[...] quem tem que ser atendido primeiro, por exemplo, o paciente que tem a cor vermelha ele é urgente, tem que atender logo; aquele paciente amarelinho pode esperar um pouco mais; laranja, verde, azul. (ACD 3)

[...] tem aquela classificação de Manchester né, a classificação de cores, que é como se fosse pra priorizar o atendimento, quem é urgente, quem corre risco de morrer, e o que não tem, que vai do vermelho até o verde. Acho que é isso. (ACD 5)

De acordo com o protocolo de Manchester, o protocolo de classificação de risco mais utilizado mundialmente dentro dos serviços de emergência, os pacientes que recebem classificação 
vermelha são pacientes considerados emergentes que deverão ser encaminhados diretamente à sala de emergência devido à necessidade de atendimento imediato.

Evidenciamos aqui uma deficiência no conhecimento dos pesquisados, como apontado pela literatura, o que se deve ao pouco ou nenhum contato que os mesmos têm com esses temas durante a sua formação. Os profissionais de enfermagem precisam estar capacitados para assistir de forma integral e qualitativa o paciente, entretanto, para que essa assistência seja de qualidade é necessário que o acadêmico, futuro profissional, seja preparado desde a graduação. $^{14}$

\section{SEGUNDA CATEGORIA}

ENTENDIMENTO ACERCA DA IMPORTÂNCIA DO ENFERMEIRO NO PROCESSO DE ACOLHIMENTO COM AVALIAÇÃO E CLASSIFICAÇÃO DE RISCO:

Nas seguintes unidades de registro podemos observar sob a óptica dos discentes a importância fundamental atribuída ao enfermeiro no acolhimento com classificação de risco.

A importância vem por conta do nosso olhar ser mais holístico, nós vemos o paciente como um todo. Nós temos uma visão mais global do atendimento, não unidirecional, para entender e atender as necessidades do usuário. Então a importância vem da visão holística que o enfermeiro tem. (ACD 1)

AH! O enfermeiro consegue ver o paciente como um todo né, ver de forma integral, ele tem conhecimento para isso. Então, ele é importante para saber classificar o paciente na urgência. (ACD 13)

É descrito que ao enfermeiro classificador cabe o papel de realizar o primeiro contato com o paciente com a finalidade de verificar prioridades de assistência à saúde, por meio da observação sistemática do mesmo, através de uma visão holística, ou seja, saber ouvir as queixas que o levaram a procurar esse serviço sejam elas físicas, psíquicas ou sociais. ${ }^{15}$

Outro fator importante que demonstra que o enfermeiro é o profissional mais indicado para o serviço de triagem é pelo fato de ter em sua formação, características generalistas, que o permitem coordenar a equipe de enfermagem, responsabilizar-se pela sua unidade de atuação, melhorar os processos de classificação de risco, encaminhando o paciente para a área clínica mais adequada.

Além do mais consegue supervisionar o fluxo de pacientes, tem autonomia sobre sua equipe, capacitando a mesma por meio da Educação Continuada; 
estimulando o espírito de liderança o que promove um melhor andamento dos serviços de triagem. ${ }^{15}$

Nestes recortes textuais fica evidente a importância do papel gerencial do profissional enfermeiro:

$O$ enfermeiro é o profissional que está mais preparado. Porque a nossa profissão já tem todo esse cunho de gerência [...] organizando e direcionando o fluxo de atendimento, dando agilidade e desafogando o serviço. (ACD 2)

[...] então, a classificação de risco acaba sendo um instrumento onde o enfermeiro pode atuar fazendo gerenciamento de pessoas, pois ele fica responsável por escolher conforme seu conhecimento qual paciente precisa de atendimento mais rápido. (ACD 6)

$O$ enfermeiro tem o olhar crítico e pode dizer com autonomia quem pode ficar esperando. Então a atuação do enfermeiro é importante para identificar e classificar de maneira correta quem precisa com mais urgência de um atendimento. (ACD 7)

$O$ enfermeiro tem o conhecimento cientifico, tem apropriação para colocar o paciente dentro de um risco correto. Ele sabe direcionar o paciente de acordo com a gravidade. $E$ se preciso, ele sabe movimentar a equipe, organizar o serviço. (ACD 8)

Logo, verifica-se que o entendimento dos pesquisados sobre a importância que o profissional enfermeiro assume na classificação de risco está de acordo com o que se encontra na literatura, onde este contribui para um melhor funcionamento do serviço de emergência, dando ordenação ao atendimento de acordo com o grau de gravidade do paciente e não por ordem de chegada, o que agiliza os processos, reduz o tempo de espera nas filas, além de proporcionar maior satisfação dos usuários dos serviços de emergência.

\section{TERCEIRA CATEGORIA}

ENTENDIMENTO ACERCA DAS ATRIBUIÇÕES DO ENFERMEIRO NO PROCESSO DE ACOLHIMENTO COM AVALIAÇÃO E CLASSIFICAÇÃO DE RISCO:

Experiências exitosas descritas na literatura sobre o acolhimento com classificação de risco têm citado o profissional Enfermeiro como o protagonista desse processo. A atuação do enfermeiro no serviço de acolhimento e classificação de risco, além de constituir uma nova área de atuação para esse profissional, possibilita melhor gerenciamento de serviços de emergência, pois contribui para garantir o acesso do paciente, diminuir o tempo de espera, diminuir os riscos e melhorar a qualidade do atendimento. ${ }^{16}$ 
Face a importância do enfermeiro nesse processo, observa-se que os pesquisados conseguem descrever, ainda que de maneira superficial, o papel do enfermeiro no processo de acolhimento com classificação de risco, como evidenciado nas falas:

Ele vai ter que conduzir o paciente para um espaço onde ele realmente possa receber um atendimento e fazer a avaliação do paciente para traçar os riscos. Além da tomada de decisões, onde o enfermeiro tem que ser ágil e hábil, tem que dominar as técnicas, a teoria e os procedimentos. (ACD 1)

Inicialmente o enfermeiro vai procurar os sinais e sintomas do paciente com auxílio dos sinais vitais, e conforme a sua avaliação e conhecimento, vai direcionar o paciente para qual tipo de atendimento deve ter, se de urgência, emergência. (ACD 2)

Ele vai acolher ouvindo as queixas, identificar possíveis sinais e sintomas presentes, fazer a anamnese, exame físico, utilizar as escalas de Glasgow e de dor, por exemplo. Fazer a avaliação do individuo quanto ao grau de gravidade, e de acordo com o protocolo de Manchester, classificar em vermelho, amarelo, verde, azul. (ACD 4) O enfermeiro precisa pensar muito rápido, ter habilidades e experiência para saber classificar o paciente. Ele deve usar seu conhecimento e ao fazer a anamnese $e$ exame físico deve classificar de acordo o protocolo de Manchester. (ACD 11)
O enfermeiro deve ser capaz de rever uma quantidade de informações, pensar criticamente, fazer julgamentos corretos e decidir que direção a avaliação deverá tomar, realizando, assim, uma coleta de dados orientada para o problema, enfocando a situação atual do cliente. Além de ser capaz de resolver problemas de forma precisa, eficaz e, algumas vezes, rápida. ${ }^{16}$

\section{QUARTA CATEGORIA}

ENTENDIMENTO ACERCA DOS PRINCIPAIS ASPECTOS QUE COLABORAM PARA A AQUISIÇÃO/NÃO AQUISIÇÃO DE HABILIDADES NO PROCESSO DE ACOLHIMENTO COM AVALIAÇÃO E CLASSIFICAÇÃO DE RISCO:

Nesta categoria, emergiram dos entrevistados informações acerca do que colaborou, o que os ajudou, para que pudessem obter o conhecimento e/ou habilidades com relação ao processo de acolhimento com avaliação e classificação de risco, e de possíveis oportunidades para a realização dessa atividade, durante a formação acadêmica.

Observou-se a prática e estágio supervisionado como os principais contribuintes para a aquisição de conhecimentos, sendo estes relacionados ao arcabouço teórico, estando diretamente 
ligados à presença e habilidades do professor-preceptor no campo.

Tal relação teoria e prática fica evidenciada nas unidades de registro:

Eu acho que o que contribuiu, principalmente, foi a questão da prática. Só com a teoria a gente fica muito restrito, $e$ não consegue visualizar essa questão do acolhimento. (ACD 1)

Acho que o que contribuiu pra eu adquirir esse conhecimento foi a própria vivência, está lá dentro, entendeu? (ACD 5)

A formação do enfermeiro deve incluir proximidade do que é ministrado em sala de aula com a prática profissional, permitindo a aproximação com questões reais do dia-a-dia para assim instrumentalizar o desenvolvimento de intervenções e pesquisas que possam produzir transformações da realidade. ${ }^{14}$

É descrito que existe uma relação de interdependência entre a teoria e a prática, que deve ser aproveitada e bem trabalhada por quem acompanha os discentes no campo. Neste contexto a teoria compõe um conjunto de conhecimentos que proporcionam ao estagiário a possibilidade de definir estratégias de ação. Ao planejarem as ações no campo prático surgem novas reflexões e conhecimentos, o que resultará no crescimento acadêmico. ${ }^{17,18}$

Outro ponto importante, mencionado pelos entrevistados, é a presença e atuação do professor-preceptor no contexto do campo de estágio, relatando ainda a importância de ser um mesmo profissional para ministrar a teoria e a prática, além da experiência deste na área em que está lotado para o estágio.

O que contribuiu foram os professores, principalmente o nosso professor do $4^{o}$ ano que foi quem nos apresentou a classificação de risco. Eu acho que foi mais mérito dos professores individualmente. Porque a gente só viu isso no $4^{o}$ ano, e às vezes se vê no $5^{\circ}$ ano muito superficial. Eu acho um pouco deficiente. (ACD 2)

É... Principalmente a participação do professor. $O$ professor ser da área! $E$ ministrar a teoria e a prática. (ACD 6)

No estágio supervisionado o preceptor necessita de competências para compartilhar conhecimentos, viável e satisfatório às demandas dos alunos. No que tange a enfermagem, questiona-se a capacidade em agregar à prática os conteúdos teóricos, isso se dá provavelmente pela desconexão e distanciamento entre a Universidade e o local de prática, assim como a desarticulação entre $o$ docente e $\quad$ o 
preceptor (professor de teoria e prática diferentes). O que resulta na incapacidade de atender as necessidades de aprendizado do aluno. ${ }^{17,18}$ Cabe ao enfermeiro preceptor do estágio supervisionado se colocar como elo entre a sua prática e os conteúdos teóricos, adequando e adaptando essa prática, utilizando de competências pedagógicas para transformar essa realidade e minimizar o distanciamento e conflito entre teoria e prática. ${ }^{19}$

Faz-se importante discutir, também, a oportunidade de realização, ou não, por parte dos acadêmicos do processo de acolhimento com avaliação e classificação de risco e quais os fatores influenciaram para isso. Foi possível observar que grande parte dos acadêmicos afirmou realizar mais a observação dessa atividade em decorrência da deficiência do campo de prática, como pode ser observado nas unidades de registro:

Sim, tive oportunidade, mas muito superficial. Nós tivemos mais uma visão geral mesmo, observacional. E foi mais esforço por parte do professor, de tentar mostrar isso pra gente. Em nenhum momento nós fizemos esse tipo de triagem com classificação. (ACD1)

Nunca realizei um acolhimento de verdade. Porque nosso campo de estagio não tinha mesmo esse acolhimento. Não tinha! (ACD 7)

Fica claro na fala dos entrevistados a falta de oportunidade para a realização concreta do acolhimento com classificação, evidenciando que esta atividade se deu em grande parte em caráter observacional. Isso reflete diretamente na formação, pois é no campo de prática que se tem a oportunidade para desenvolver competências e habilidades importantes para a formação, onde este pode (ou pelo menos poderia) vivenciar a realidade do processo de trabalho do enfermeiro nos vários contextos de atuação.

Há um anseio por uma formação qualificada, com experiências práticas, que venham contemplar o aluno em sua totalidade. ${ }^{20}$ Portanto, em meio a um cenário de mudanças e exigências crescentes, ainda se observa que a articulação teórico-prática não ocorre satisfatoriamente, sendo explorada de forma superficial ao longo do processo de formação do Enfermeiro. Tal distanciamento entre teoria e prática pode ser percebido quando o profissional recémformado depara-se com situações que lhe parecem absolutamente novas, visto que não foram vivenciadas durante sua 
formação ou o foram vivenciadas de modo distinto da realidade profissional. ${ }^{19,20}$

\section{CONCLUSÃO}

Através dos dados encontrados neste estudo, foi possível descrever o saber de um grupo de acadêmicos de Enfermagem de uma IES da cidade de Belém, Estado do Pará. A prática do Acolhimento com Avaliação e Classificação de Risco na óptica dos acadêmicos emergiu como uma metodologia de fundamental importância, pois se trata de um processo dinâmico de identificação e priorização do atendimento, o qual visa discernir os casos críticos que necessitam de atendimento imediato dos não críticos.

A temática deve ser melhor abordada na formação em saúde, com um enfoque nas questões fisiológicas, deontológicas e éticas envolvidas no processo. $\mathrm{O}$ distanciamento entre o que é ensinado nos cursos de Graduação em Enfermagem e aquilo que o profissional vivencia em sua prática pode ser confirmado pela literatura e pelos achados deste estudo.

Embora a IES que serve de cenário ao estudo apresente avanços inquestionáveis em relação à inclusão do tema na Graduação em Enfermagem, atenta-se para as fragilidades evidenciadas nas dimensões estruturais e recursos humanos, uma vez que, provavelmente, interfiram negativamente na construção de conhecimentos sólidos acerca da temática.

Ressalta-se a importância da formação profissional para o atendimento no processo de acolhimento com classificação de risco. Porém, fica evidente o distanciamento entre o que é ensinado no curso de Graduação em Enfermagem e aquilo que o profissional vivencia em sua prática. Cabe ao ensino prático curricular contribuir para a formação de forma que ultrapasse a troca de saberes, com o compromisso de desenvolver com a ajuda das competências pedagógicas do professor-preceptor, um profissional que esteja em contato com a realidade de sua futura profissão, de modo que possa associar além da teoria e prática, conteúdos, intencionalidade e condições para a ação.

A conclusão da graduação é muito significativa, no entanto é motivo de grande angústia e ansiedade. Comumente, o primeiro emprego é permeado por desafios que acompanham o Enfermeiro em seus primeiros meses de exercício profissional. Este enfrenta a insegurança e receios, diante de inúmeras dificuldades, 
que vão desde o processo admissional até a adaptação às normas e ao processo de trabalho da instituição. Dentre as possíveis causas da predominância dessas dificuldades, destaca-se a falta de oportunidades no campo prático, durante a Graduação, que em grande parte não oferecem a oportunidade de desenvolver todas as habilidades e competências necessárias para a atuação profissional.

É imprescindível atentar-se à realidade do ensino em enfermagem do país. A formação acadêmica deve permitir uma visão crítica e reflexiva da profissão, mostrando a importância do papel social e político do Enfermeiro na assistência, no gerenciamento, no ensino, na pesquisa, a fim de valorizar o profissional e alcançar melhores condições de trabalho. Portanto, o estudo implica em repensar o processo de formação, com ênfase na articulação de conteúdos do currículo, bem como na articulação teórico-prática que favoreça transformações compartilhadas dos serviços e da instituição, favorecendo também a formação de um profissional capaz de atuar como promotor da saúde integral do ser humano, nos seus diversos campos de atuação.
O estudo apresenta como limitação seu aspecto regional, tornando limitada a possibilidade de generalizações. Diante do exposto, esta pesquisa suscita estudos futuros para que se quantifiquem os impactos da sujeição do processo aos entraves apontados.

\section{REFERÊNCIAS}

1. Paixão TCR, Campanharo CRV, Lopes MCBT, Okuno MFP, Batista REA. Dimensionamento de enfermagem em sala de emergência de um hospital-escola. Rev Esc Enferm USP [internet]. 2015 [citado em 01 jul 2016]; 49(3): 486-493. Disponível em: http://www.scielo.br/pdf/reeusp/v49n3/pt_ 0080-6234-reeusp-49-03-0486.pdf

2. Versa GLGS, Vituri DWV, Buriola AA, Oliveira CA, Matsuda LM. Avaliação do acolhimento com classificação de risco em serviços de emergência hospitalar. Rev Gaúcha Enferm [internet]. 2014 [citado em 30 jun 2016]; 35(3): 21-28. Disponível em: http://www.scielo.br/pdf/rgenf/v35n3/pt_1 983-1447-rgenf-35-03-00021.pdf

3. Oliveira DA, Guimarães JP. A importância do acolhimento com Classificação de risco nos serviços de Emergência. Caderno Saúde e Desenvolvimento [internet]. 2013 [citado em 02 jul 2016]; 2(2): 25-44. Disponível em:

http://www.uninter.com/revistasaude/index .php/cadernosaudedesenvolvimento/article/ viewFile/197/101

4. Inoue KC, Bellucci Júnior JA, Papa MAF, Vidor RC, Matsuda LM. Avaliação da qualidade da Classificação de Risco nos Serviços de Emergência. Acta Paul Enferm [internet]. 2015 [citado em 01 jul 2016]; 
28(5): 420-425. Disponível em: http://www.scielo.br/pdf/ape/v28n5/19820194-ape-28-05-0420.pdf

5. Acosta AM, Duro CLM, Lima MADS. Atividades do enfermeiro nos sistemas de triagem/classificação de risco nos serviços de urgência: revisão integrativa. Rev Gaúcha Enferm [internet]. 2012 [citado em 01 jul 2016]; 33(4): 181-190. Disponível em:

http://www.scielo.br/pdf/rgenf/v33n4/23.p df

6. Oliveira KKD; Amorim KKPS; Fernandes APNL; Monteiro AI. Impacto da implementação do acolhimento com classificação de risco para o trabalho dos profissionais de uma unidade de pronto atendimento. Rev Min Enferm [internet]. 2013 [citado em 01 jul 2016]; 17(1): 157$164 . \quad$ Disponível em: http://www.reme.org.br/artigo/detalhes/586 7. Guedes MVC, Henriques ACPT, Lima MMN. Acolhimento em um serviço de emergência: percepção dos usuários. Rev Bras Enferm [internet]. 2013 [citado em 30 jun 2016]; 66(1): 31-37. Disponível em: http://www.scielo.br/pdf/reben/v66n1/v66n 1a05.pdf

8. Oliveira LV, Leite NL, Cavalcante CAA, Miranda FAN. O cuidar de presidiários sob a ótica de acadêmicos de enfermagem. J. Res. Fundam. Care [internet]. 2016 [citado em 03 jul 2016]; 8(1): 3780-3792. Disponível em: http://www.seer.unirio.br/index.php/cuidad ofundamental/article/viewFile/4015/pdf_1 790

9. Zanatta JA, Costa ML. Algumas reflexões sobre a pesquisa qualitativa nas ciências sociais. Estud. Pesqui. Psicol [internet]. 2012 [citado em 02 jul 2016]; 12(2): 344-359. Disponível em: http://www.redalyc.org/articulo.oa?id=451 844638002
10. Fontanella BJB, Magdaleno Júnior R. Saturação teórica em pesquisas qualitativas: contribuições psicanalíticas. Psicol em Estud, Maringá [internet]. 2012 [citado em 02 jul 2016]; 17(1): 63-71. Disponível em: http://www.scielo.br/pdf/pe/v17n1/v17n1a 07.pdf

11. Cavalcante RB, Calixto P, Pinheiro MMK. Análise de conteúdo: considerações gerais, relações com a pergunta de pesquisa, possibilidades e limitações do método. Inf. \& Soc. Est. [internet]. 2014 [citado em 03 jul 2016]; 24(1): 13-18. Disponível em: http://www.ies.ufpb.br/ojs/index.php/ies/ar ticle/view/10000/10871

12. Câmara RH. Análise de conteúdo: da teoria à prática em pesquisas sociais aplicadas as organizações. Rev Interinstit de Psicol [internet]. 2013 [citado em 03 jul 2016]; 6(2): 179-191. Disponível em: http://pepsic.bvsalud.org/pdf/gerais/v6n2/v 6n2a03.pdf

13. Fernandes FSL , Lima BSL, Ribeiro MN. Welcoming with Risk Classification in the Hospital São Paulo's Emergency Department. Acta Paul Enferm [internet]. 2012 [citado em 02 jul 2016]; 25 (Special Issue 2): 164-168. Disponível em: http://www.scielo.br/pdf/ape/v25nspe2/26. pdf

14. Trevisan DD, Minzon DT, Testi CV, Ramos NA, Carmona EV, Silva EM. Formação de enfermeiros: distanciamento entre a graduação e a prática profissional. Cienc Cuid Saúd [internet]. 2013 [citado em 03 jul 2016]; 12(2): 331-337. Disponível em: http://www.periodicos.uem.br/ojs/index.ph p/CiencCuidSaude/article/viewFile/19643/ pdf

15. Souza CC, Diniz AS, Silva LLT, Mata LRF, Chianca TCM. Nurses' perception about risk classification in an 
emergency service. Investigación y Educación en Enfermería [internet]. 2016; [citado em 03 jul 2016]; 32(1): 78-86. Disponível em: http://www.redalyc.org/articulo.oa?id=105 230027009

16. Camara RF, Paulino TS, Pereira FCCP, Nelson ICASR, Rocha KM, Inácio Neto L. O papel do enfermeiro no processo de classificação de risco na urgência: uma revisão. Revista Humano Ser UNIFACEX [internet]. 2015 [citado em 03 jul 2016]; 1(1): 99-114. Disponível em: https://periodicos.unifacex.com.br/humano ser/article/view/628/146

17. Almeida EB, Lüdke M. O estágio como espaço de reflexão entre a teoria e a prática. Rev Intersab [internet]. 2012; 7(14): 429-433. Disponível em: http://www.uninter.com/intersaberes/index. php/revista/article/view/334/205

18. Moreira F, Ferreira E. Teoria, prática e relação na formação inicial na enfermagem e na docência. Educ Socied \& Cult [internet]. 2014 [citado em 02 jul 2016]; 41: 127-148. Disponível em: http://www.fpce.up.pt/ciie/sites/default/file s/ESC41_F_Moreira_E_Ferreira.pdf 19. Rodrigues LMS, Tavares CMM. Estágio supervisionado de enfermagem na atenção básica: o planejamento dialógico como dispositivo do processo ensinoaprendizagem. Rev Rene [internet]. 2012 [citado em 02 jul 2016]; 13(5): 1075-1083. Disponível em: http://www.revistarene.ufc.br/revista/index .php/revista/article/viewFile/62/pdf

20. Oliveira AG. Estágio supervisionado em enfermagem: visão de preceptores. [dissertação]. Natal (RN): Universidade Federal do Rio Grande do Norte/UFRG; 2014. 82 p.
Recebido em 14/02/2016

Aprovado em 22/12/2016

Publicado em 29/12/2016 